\title{
In silico genome analysis reveals the metabolic versatility and biotechnology potential of a halotorelant phthalic acid esters degrading Gordonia alkanivorans strain YC-RL2
}

\author{
Ruth Nahurira, Junhuan Wang, Yanchun Yan*, Yang Jia, Shuanghu Fan, Ibatsam Khokhar and Adel Eltoukhy
}

\begin{abstract}
Members of genus Gordonia are known to degrade various xenobitics and produce secondary metabolites. The genome of a halotorelant phthalic acid ester (PAEs) degrading actinobacterium Gordonia alkanivorans strain YC-RL2 was sequenced using Biosciences RS II platform and Single Molecular Real-Time (SMRT) technology. The reads were assembled de novo by hierarchical genome assembly process (HGAP) algorithm version 2. Genes were annotated by NCBI Prokaryotic Genome Annotation Pipeline. The generated genome sequence was 4,979,656 bp with an average $\mathrm{G}+\mathrm{C}$ content of $67.45 \%$. Calculation of ANI confirmed previous classification that strain YC-RL2 is G. alkanivorans. The sequences were searched against KEGG and COG databases; 3132 CDSs were assigned to COG families and 1808 CDSs were predicted to be involved in 111 pathways. 95 of the KEGG annotated genes were predicted to be involved in the degradation of xenobiotics. A phthalate degradation operon could not be identified in the genome indicating that strain YC-RL2 possesses a novel way of phthalate degradation. A total of 203 and 22 CDSs were annotated as esterase/hydrolase and dioxygenase genes respectively. A total of 53 biosynthetic gene clusters (BGCs) were predicted by antiSMASH (antibiotics \& Secondary Metabolite Analysis Shell) bacterial version 4.0. The genome also contained putative genes for heavy metal metabolism. The strain could tolerate $1 \mathrm{mM}$ of $\mathrm{Cd}^{2+}, \mathrm{Co}^{2+}, \mathrm{Cu}^{2+}, \mathrm{Ni}^{2+}, \mathrm{Zn}^{2+}$, $\mathrm{Mn}^{2+}$ and $\mathrm{Pb}^{2+}$ ions. These results show that strain YC-RL2 has a great potential to degrade various xenobiotics in different environments and will provide a rich genetic resource for further biotechnological and remediation studies.
\end{abstract}

Keywords: Gordonia, Aromatic pollutants, Secondary metabolites, Heavy metals, Phthalic acid esters

\section{Introduction}

Environmental pollution has become a global concern that requires concerted effort for monitoring, control and remediation plans across all sectors of society. Industrialization and urban development, advanced and commercial agriculture have all accelerated pollution. Recalcitrant organic xenobiotic compounds such as polychlorinated biphenyls (PCBs), plasticizers, synthetic polymers have attracted attention not only due to their persistence in the environment but all also their toxicity (El-Shahawi et al. 2010; Schäfer et al. 2011). Metal

*Correspondence: yanyanchun@caas.cn

Biological Laboratory, Department of Biology, Graduate School of Chinese Academy of Agricultural Sciences, Beijing, People's Republic of China pollution is another concern for terrestrial and aquatic ecosystems (Fu and Wang 2011). Metals are naturally occurring in earth's crust but anthropogenic activities have resulted in elevated levels that are detrimental to life. Metals at the right concentrations play crucial roles in biochemical and physiological activities but elevated levels can lead to an equilibrium shift leading to abnormalities and disease (Tchounwou et al. 2012; Wang and Shi 2001). Therefore, there is an urgent need for bioremediation and microbes have a rich potential for remediation of contaminated sites and synthesis of secondary metabolites that would be eco-friendly alternatives to their synthetic congeners (Das and Chandran 2011; Megharaj et al. 2011). 
Members of phylum Actinobacteria possess a great potential for remediation of contaminated environments through degradation of xenobiotics, sequestration of heavy metals and production of novel secondary metabolites. In the phylum Actinobacteria, the genus of Gordonia has recently gained attention due to the biotechnological potential of its members (Drzyzga 2012). Members of genus Gordonia are high $\mathrm{G}+\mathrm{C}$ gram positive bacteria of the order Actinomycetales (Arenskötter et al. 2004). Their ability includes but is not limited to degradation of various phthalic acid esters (PAEs), poly aromatic hydrocarbons (PAHs), alkylpyridines, 1,3,5-triazines and synthetic isoprene rubber (Drzyzga 2012). Production of extracellular polysaccharides (Ta-Chen et al. 2008), carotenoids (Arenskötter et al. 2004), steroids (Schneider et al. 2008), and surfactants (Drzyzga 2012) has been documented. With major technological advances in genome sequencing, prediction and elucidation of the biosynthetic pathways of these novel compounds through genome mining is now possible. Varied microbial natural products such as polyketide synthases (PKSs, NRPSs, and the ribosomally synthesized and post-translationally modified peptides (RiPPs) (Letzel et al. 2014; Liu et al. 2016) have recently gained attention in the pharmaceutical industry. Most Gordonia species contain genes for polyketide and carotenoid biosynthesis and alkane degradation (Sowani et al. 2018).

A halotorelant PAEs degrading Gordonia alkanivorans strain YC-RL2 was previously isolated from contaminated soil. It had an outstanding ability to utilize PAEs and other aromatic compounds as the sole carbon and energy source in varied environmental conditions (Nahurira et al. 2017). Strain YC-RL2 could transform dialkyl phthalates (DAPs) into phthalate (PA) via monoalky phthalates (MAPs) and then to benzoic acid (BA). Typically, the biodegradation process of PAEs is initiated by the hydrolysis of ester linkage forming monoester and subsequently PA and then protocatechuate. Though PA degrading genes are well characterized, not so much is known about genes and enzymes involved in the hydrolysis of PAEs to PA and its transformation to benzoate under aerobic conditions. Furthermore, not much is known about the genetic basis of YC-RL2 outstanding ability to utilize diversified substrates and adapt to a wide range of environmental conditions. This study focused on analyzing genome information to unveil the underlying genetic information of strain YC-RL2's ability to degrade various aromatic compounds, PAEs, synthesize secondary metabolites and survive in contaminated environments.

\section{Materials and methods}

\section{Chemicals and media}

Chemicals used in this study were all of analytical grade. Diphenyl ether, napthol, benzene, para-chlorobenzoic acid (PCBA), triphenyl phosphate (TPhP) and phenyl phosphate were purchased from Shanghai Macklin Biochemical Co., Ltd (Shanghai, China). Dichloromethane $\left(\mathrm{CHCl}_{2}\right), o$-xylene, phenol, hexane and naphthalene were Sinopharm Chemical reagent Co., Ltd (Beijing, China). Tetra chlorobenzene and $\mathrm{CHCl}_{3}$ were from Beijing Chemical works Ltd while cholesterol was obtained from Beijing Solarbio life technology Co., Ltd (Beijing, China). Biphenyl, PCB No. 1 (2-Chlorobiphenyl), PCB No. 11 (3, 3'-Dichlorobiphenyl), PCB No.31 (2,4',5-Trichlorobiphenyl), PCB No. 47 (2,2',4,4'-Tetrachlorobiphenyl) were obtained as standards from Dr. Ehrnstorfer GmbH Laboratories (Augsburg-Germany).

\section{Substrate utilization tests and intermediate analysis}

The ability of strain YC-RL2 to utilize diphenyl ether, naphthol, $\mathrm{CHCl}_{2}$, benzene, phenol, PCBA, TPhP, $o$-xylene, hexane, tetra chlorobenzene, cholesterol, naphthalene, $\mathrm{CHCl}_{3}$ and phenyl phosphate, biphenyl and PCBs was tested by inoculating the strain in trace element medium (TEM) medium supplemented with $50 \mathrm{ppm}$ of each. Each experiment was setup in triplicate and the setup without inoculum acted as a control treatment. Cell growth was measured with UV-Vis spectrometer (Biomate 3S-Thermoscientific) as optical density at $600 \mathrm{~nm}$ after 7 days.

\section{DNA extraction, genome library preparation and sequencing}

The strain was previously isolated from petroleum contaminated soil as described before (Nahurira et al. 2017). It was cultured in LB liquid medium for 4 days at $30{ }^{\circ} \mathrm{C}$ and $180 \mathrm{rpm}$ until the $\mathrm{OD}_{600}$ reached 1.0. This culture was then used for DNA extraction.

The genomic DNA was extracted using Qiagen QIAamp DNA Kit according to manufacturer's instructions. The size, quantity and quality of the DNA were checked by Agilent 2100 Bioanalyzer (Santa Clara, CA, USA). The genome was then sequenced by Pacific Biosciences RS II platform and Single Molecular RealTime (SMRT) technology (Ardui et al. 2018; Shin et al. 2013). The raw sequence data were filtered, and 186,554 high quality reads were obtained with a total of $1,912,577,568 \mathrm{bp}$ and average reads length of 10,252 bp. The reads were assembled de novo by applying the hierarchical genome assembly process (HGAP) algorithm version 2 (Jayakumar and Sakakibara 2017). Prediction and annotation of genes, protein coding sequences, tRNA and rRNA carried out using by NCBI Prokaryotic 
Genome Annotation Pipeline (https://www.ncbi.nlm.nih. gov/genome/annotation_prok/).

\section{Phylogenetic and comparative genomic analysis}

Strain YC-RL2 was originally classified as G. alkanivorans based on the partial sequence of 16S rRNA gene sequence (KR819397.1). To confirm its classification status, the genome was used to calculate Average Nucleotide Identity (ANI) values. ANI values among Gordonia strains were calculated by JSpecies (http://jspecies.riboh ost.com/jspeciesws/) equipped with local access to BLAST + and MUMmer. Genome-derived 16S rRNA gene sequence was also used to ascertain its phylogenetic relationship with Gordonia strains validly published at LSPN bacterio.net. The sequences were imported to MEGA 6.0 software (Tamura et al. 2013) and analyzed by neighbor-joining algorithm with 1000 bootstrap values.

Genome annotated dioxygenases were searched against SMART (http://smart.embl-heidelberg.de/), interproscan (https://www.ebi.ac.uk/interpro/) and conserved domain database (CDD) (https://www.ncbi.nlm.nih.gov/cdd) to check for the resemblance of domains and filter out false positives. The filtered putative dioxygenases sequences were then aligned using blastP with sequences from swissprot and pdb databases. The alignments producing Expect (E) values of $<10^{-10}$ were selected for further analysis. In case of alignments with values of $>10^{-10}$, percentage identity of $30 \%$ was used as a criterion. If neither of the above were satisfied, sequences of bitscores of $>50$ were considered. The protein sequences were retrieved from NCBI in FASTA format and phylogenetic trees were constructed by neighbor-joining algorithms in the MEGA 6.0 program.

All CDS annotated as esterases/carboxylesterases and hydrolases were compared to the phthalate hydrolases in literature by examining their conserved domains (https:// www.ncbi.nlm.nih.gov/cdd) and motifs. The sequences were searched for the signature pentapeptide (GXSXG) and HGG motif that are characteristic of esterases (Hausmann and Jaeger 2010).

\section{Prediction of secondary metabolite potential}

To predict anabolic potential of strain YC-RL2, putative biosynthetic gene clusters (BGCs) were annotated by antiSMASH (antibiotics \& Secondary Metabolite Analysis Shell 4.0) (Weber et al. 2018).

\section{Heavy metal tolerance tests}

Heavy metal tolerance was determined by growing the strain aerobically in stationary 96 well plates in LB medium supplemented with appropriate concentration of heavy metals. The growth of the strain was measured by monitoring the changes in the optical density $\left(\mathrm{OD}_{600}\right)$ using an infinite M200PRO (TECAN) plate reader every $24 \mathrm{~h}$ for 4 days. All experiments were conducted in triplicate. The strain was tested for growth in divalent heavy metals such as $1 \mathrm{mM}$ of $\mathrm{Cd}^{2+}, \mathrm{Co}^{2+}, \mathrm{Cu}^{2+}, \mathrm{Ni}^{2+}, \mathrm{Zn}^{2+}$, $\mathrm{Mn}^{2+}$ and $\mathrm{Pb}^{2+}$ ions and $0.1 \mathrm{mM}$ of $\mathrm{Hg}^{2+}$. A growth of $\mathrm{OD}>0.2$ was considered as metal resistant for that treatment (Uhrynowski et al. 2017).

\section{Accession numbers}

Strain YC-RL2 has been deposited in China General Microbiological Culture Collection Center (CGMCC) under accession number CGMCC 10992. The complete chromosome and plasmid sequences of G. alkanivorans strain YC-RL2 were deposited in GenBank database under accession number CP027114 and CP027115 respectively. The BioProject and BioSample information is available at PRJNA434635 and SAMN08565628 respectively.

\section{Results}

\section{Genome sequence and annotation results}

The generated genome sequence of strain YC-RL2 comprised of 4,979,656 bp with an average $\mathrm{G}+\mathrm{C}$ content of $67.45 \%$. The sequence was assembled into one circular chromosome $(4,921,996 \mathrm{bp}$ and one plasmid (pYC01, $57,660 \mathrm{bp}$ ). In total, 4541 genes were predicted, including 4308 protein coding sequences with 63 rRNA genes. More genome features are presented in Table 1.

\section{Classification}

The strain was originally classified as G. alkanivorans strain YC-RL2 based on its partial 16S rRNA gene sequence (KR819397.1). With the genome results,

\section{Table 1 A table showing genome features of YC-RL2}

\begin{tabular}{ll}
\hline Gene features & Number/comment \\
\hline Genes (total) & 4541 \\
CDS & 4478 \\
CDS (coding) & 4308 \\
Genes (RNA) & 63 \\
RNA genes & 63 \\
rRNAs & $4,4,4(5 S, 16 \mathrm{~S}, 23 \mathrm{~S})$ \\
tRNAs & 48 \\
ncRNAs & 3 \\
Pseudo genes (total) & 170 \\
Pseudo genes (ambiguous residues) & 0 of 170 \\
Pseudo genes (frame shifted) & 79 of 170 \\
Pseudo genes (incomplete) & 97 of 170 \\
Pseudo genes (internal stop) & 20 of 170 \\
Pseudo genes (multiple problems) & 24 of 170 \\
\hline
\end{tabular}


we aligned the 16S rRNA gene sequence against the 39 Gordonia strains validly published at LSPN bacterio.net. The strain YC-RL2 was closely related with $G$. nitida (AF148947.1) though clustered together with $G$. alkanivorans (Y18054.1) (Additional file 1: Figure S1). Using BLAST, the complete 16S rRNA gene sequence of strain YC-RL2 showed 100\% identity with 16S rRNA gene sequence of AB065369.1, G. alkanivorans strain CC-JG39 (AY864338.1), Gordonia sp. CNJ863 PL04 (DQ448700.1) and G. alkanivorans strain DSM 44187 (AY995556.1).

We therefore calculated the average nucleotide identity (ANI) with JSpecies (http://jspecies.ribohost.com/jspec iesws/). ANI is a similarity index between a given pair of genomes that can be applicable to prokaryotic organisms independently of their $\mathrm{G}+\mathrm{C}$ content. The calculated ANIb, ANIm and Pearson correlation coefficient values of strain YC-RL2 and G. alkanivorans NBRC 16433 and G. alkanivorans CGMCC 6845 were: $97.03 \%$ and $96.71 \%$, $97.91 \%$ and $97.56 \%, 0.99974$ and 0.99964 respectively. Though G. rubripertincta NBRC 101908 shows a Pearson correlation coefficient greater than the cutoff of 0.999 , its ANib and ANIm results were below the cut-off of $95 \%$ (Table 2). These findings support the initial classification that strain YC-RL2 belongs to G. alkanivorans species according to cut-off values for species differentiation (95-96\% for ANI).

\section{COG analysis and KEGG analysis}

The CDSs were searched against the KEGG and COG databases to investigate the functions of the genes and metabolic pathways they are involved in (Kanehisa et al.
2018). 3132 CDSs were allocated to COG families and 1808 CDSs were involved in 111 pathways.

The database of Clusters of Orthologous Groups of proteins (COGs) phylogenetically classifies proteins (http:// www.ncbi.nlm.nih.gov/COG) by applying an exhaustive comparison of all protein sequences from genomes (Tatusov 2000). The result of COG analysis of YC-RL2 as shown in Fig. 1 and the circular representation of the genome was further visualized by Circos (Krzywinski et al. 2009) as displayed in Additional file 1: Figure S2. The COG database provides a platform to assess evolutionary-oriented relationships of protein families. A large portion of CDS (1394) were not annotated to any COG groups.

95 of the KEGG annotated genes were predicted to be involved in the degradation of xenobiotics such as chloroalkanes, chloroalkenes, chlorobenzene, benzoate, flourobenzoate, dioxins, $o$-xylene, toluene, naphthalene, styrene and atrazine. 26 more genes were annotated to be involved in aromatic compound degradation.

From the KEGG prediction, we tested the ability of strain YC-RL2 to utilize diphenyl ether, naphthol, dichloromethane, benzene, phenol, PCBA, tri phosphate (TPhP), o-xylene, hexane, tetra chlorobenzene, cholesterol, naphthalene, $\mathrm{CHCl}_{3}$ and phenyl phosphate as the sole carbon and energy source and the results are shown in the Fig. 2. The cell growth was measured with UVVis spectrometer as optical density at $600 \mathrm{~nm}$. We also tested the strain's ability to degrade various PCBs and the results are shown in Fig. 3. To the best of our knowledge, this is the first report of a Gordonia species to degrade PCBs. The position and number of chlorine substitutions

Table 2 A table showing ANI values as calculated by ANIb and ANIm

\begin{tabular}{|c|c|c|c|}
\hline Genome & ANIb [\%] & ANIm [\%] & $\begin{array}{l}\text { Pearson } \\
\text { correlation } \\
\text { coefficient }\end{array}$ \\
\hline Gordonia alkanivorans NBRC 16433 & 97.03 & 97.91 & 0.99974 \\
\hline Gordonia alkanivorans CGMCC 6845 & 96.71 & 97.56 & 0.99964 \\
\hline Gordonia rubripertincta NBRC 101908 & 91.85 & 92.66 & 0.99933 \\
\hline Gordonia namibiensis NBRC 108229 & 91.29 & 92.39 & 0.99896 \\
\hline Gordonia paraffinivorans NBRC 108238 & 82.21 & 86.1 & 0.99402 \\
\hline Gordonia sp. KTR9 & 80.62 & 85.79 & 0.9925 \\
\hline Gordonia terrae NBRC 100016 & 80.48 & 85.71 & 0.98842 \\
\hline Gordonia bronchialis DSM 43247 & 76.56 & 84.95 & 0.97643 \\
\hline Gordonia soli NBRC 108243 & 75.21 & 84.68 & 0.96966 \\
\hline Gordonia amarae NBRC 15530 & 74.95 & 84.67 & 0.95839 \\
\hline Gordonia sputi NBRC 100414 & 74.62 & 84.6 & 0.95702 \\
\hline Gordonia aichiensis NBRC 108223 & 74.35 & 84.6 & 0.94806 \\
\hline Gordonia sp. QH-11 & 73.31 & 84.55 & 0.92141 \\
\hline Gordonia sihwensis NBRC 108236 & 73.08 & 84.44 & 0.90709 \\
\hline
\end{tabular}

Italicized values show results above the cut-off points 


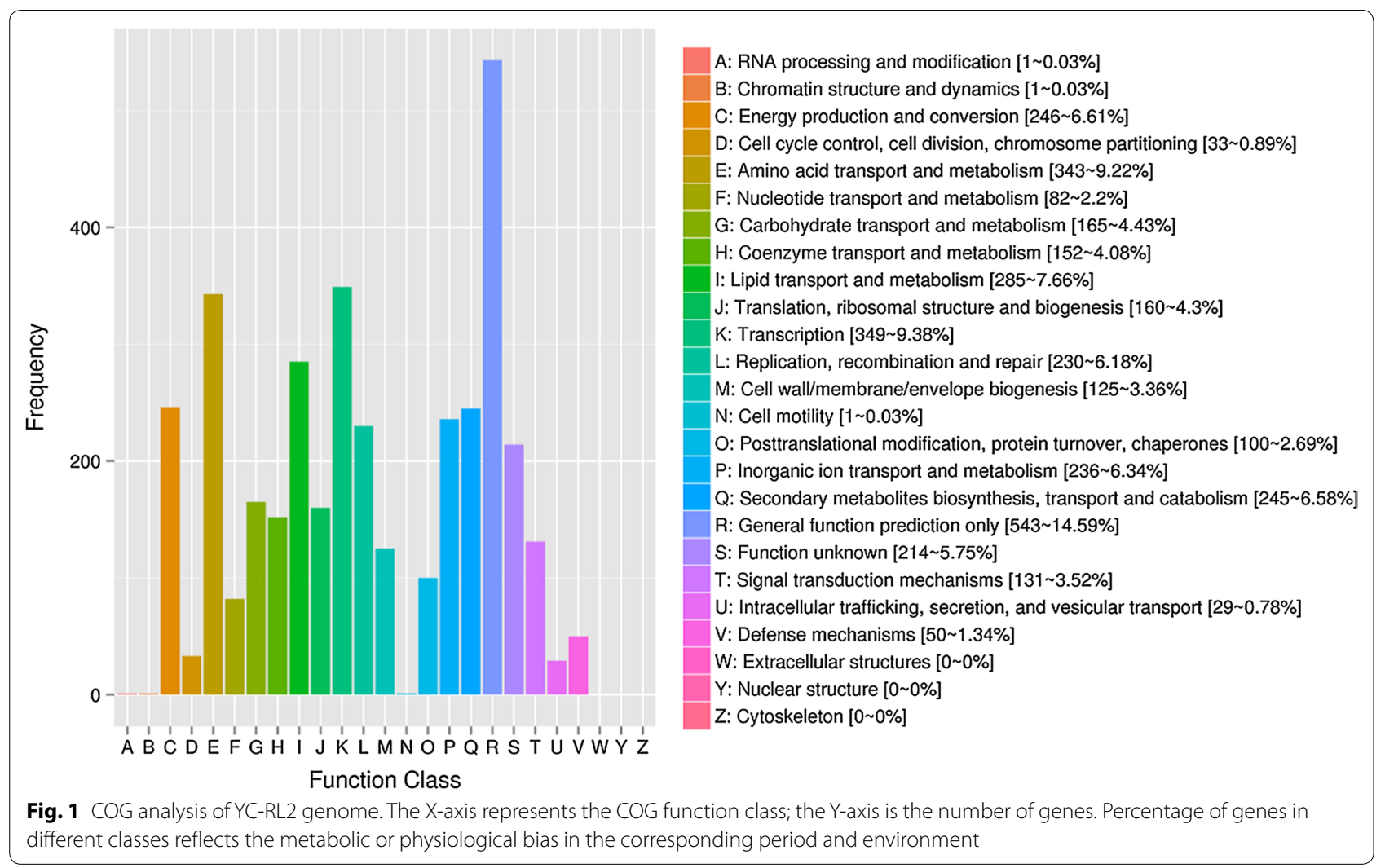

affect biodegradability of PCBs. Strain YC-RL2 could easily degrade biphenyl, PCB-1 and PCB-11 but couldn't degrade PCB-31, PCB-47 and other highly substituted PCBs.

\section{Analysis of phthalate catabolic genes Putative PAEs esterases}

A total of 203 CDS were annotated as esterases or hydrolases in the genome of strain YC-RL2. Esterases hydrolyze a broad spectrum of substrates (Ren et al. 2016), demonstrating that strain has great potential for degrading various xenobiotics.

We compared genome derived esterases/hydrolase sequences to those of reported PAEs hydrolases in literature. We chose those that shared conserved domains with the reported hydrolases. Phthalate hydrolases in literature have para-nitrobenzyl (PnbA), pimeloyl-methyl ester esterase (MhpC), acetyl ester (Aes), $N$-Carbamoylsarcosine amidohydrolase (CSHase) and $\beta$-lactamase domains. The genome of strain YC-RL2 possesses 2 PnbA, 20 $\mathrm{MhpC}, 3$ Aes and $3 \beta$-lactamase domain-containing putative esterases. The MhpC domain was found in monoethylhexyl phthalate hydrolases (Iwata et al. 2015; Nahurira et al. 2018) and the other domains in DAP hydrolases. A monoethylhexyl phthalate hydrolase $(\mathrm{MehpH})$ was cloned from strain YC-RL2 and characterized (Nahurira et al. 2018). This enzyme was able to convert MAPs to PA. Eight putative esterases were considered for further analysis (denoted by Refseq accessions of protein product as annotated by NCBI). Though they shared conserved domains with the reported DAP hydrolases, they showed low identity: WP_006357554.1 shared 26\% with CarEW from Bacillus sp. K91(Ding et al. 2015), WP_005199300.1 and WP_006868835.1 showed 26\% and 32\% respectively with Est1 from Sulfobacillus acidophilus (Zhang et al. 2014), WP_005200335.1, WP_006358922.1 and WP_005200181.1 shared 26\%, 27\% and 38\% respectively with EstG from Sphingobium sp. SM42 (Whangsuk et al. 2015), WP_006358366.1 and WP_006358508.1 shared $35 \%$ and 37\% with CarEW from Bacillus sp. K91 respectively. A phylogenetic tree showing the relationship of the putative esterases/hydrolases and reported DAP hydrolases is shown in Fig. 4.

\section{Putative decarboxylases}

Analysis of PAEs degradation intermediates showed that mono ethylhexyl phthalate (MEHP), PA and benzoic acid (BA) were the major intermediates (Nahurira et al. 2017). We therefore hypothesized that PA could be decarboxylated to BA. Ebenau-Jehle et al. (2017) reported the role of phthalate and succinyl-CoA-specific CoA transferase and a UbiD-like phthaloyl-CoA 


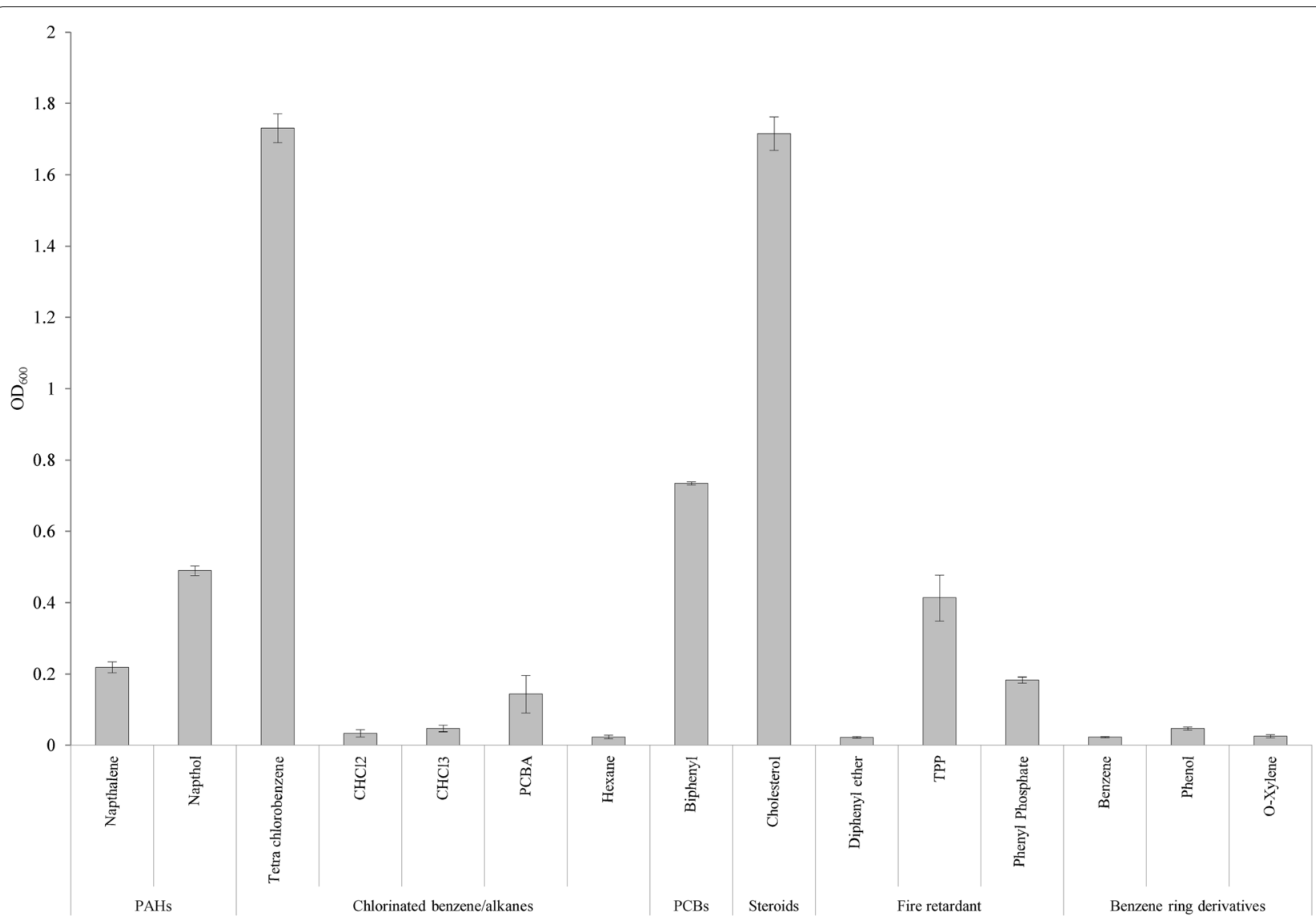

Fig. 2 A figure showing the growth of YC-RL2 when inoculated with different substrates as sole carbon and energy source. The $O_{600}$ measurements were taken after 7 days of incubation. The experimental set up consisted of TEM medium with 1\% (VN) of YC-RL2 inoculum and appropriate amount of substrates. The abiotic setup was used as a control. For each setup, it was conducted in triplicate and errors show standard deviation from the mean value

decarboxylase in anaerobic phthalate degradation (Ebenau-Jehle et al. 2017). Zhao et al. (2018) also predicted that an aerobic bacterium, Rhodococcus sp. 2G, could decarboxylate PA to BA through phthaloyl-CoA and finally to benzoyl-CoA (Zhao et al. 2018). We therefore searched phthaloyl-CoA decarboxylase and succinyl-CoA and phthalate-specific CoA transferase sequences against the genome of YC-RL2. However, there were no decarboxylases that shared similarity to those reported. This is not surprising because strain YC-RL2 is an obligate aerobe and is most likely to lack anaerobic enzymes.

However, a total of 16 decarboxylases were predicted in the genome of YC-RL2 and these were annotated as aspartate, carboxymuconolactone, acetoacetate, threonine-phosphate, uroporphyrinogen, orotidine-5'phosphate, bifunctional phosphopantothenoylcysteine, diaminopimelate, multifunctional oxoglutarate and OHCU decarboxylases.

\section{Benzoate catabolism operon}

Analysis of YC-RL2 genome sequence revealed several gene clusters involved in degradation of organic compounds. Complete operons involved in the degradation of biphenyl, benzoate and catechol were annotated in the genome. Analysis of the putative benzoate operon by Rapid Annotation using Subsystems Technology (RAST) version 2.0 (Aziz et al. 2008; Brettin et al. 2015) revealed that the deduced amino acid sequences encoded by the YC-RL2 benzoate catabolic genes are arranged in the order ben $A B C D$ (Fig. 5). The genes ben $C$ and benD are fused together with a C-terminal ligand binding (FCD) domain (Additional file 1: Figure S3) characteristic of GntR regulatory proteins. 


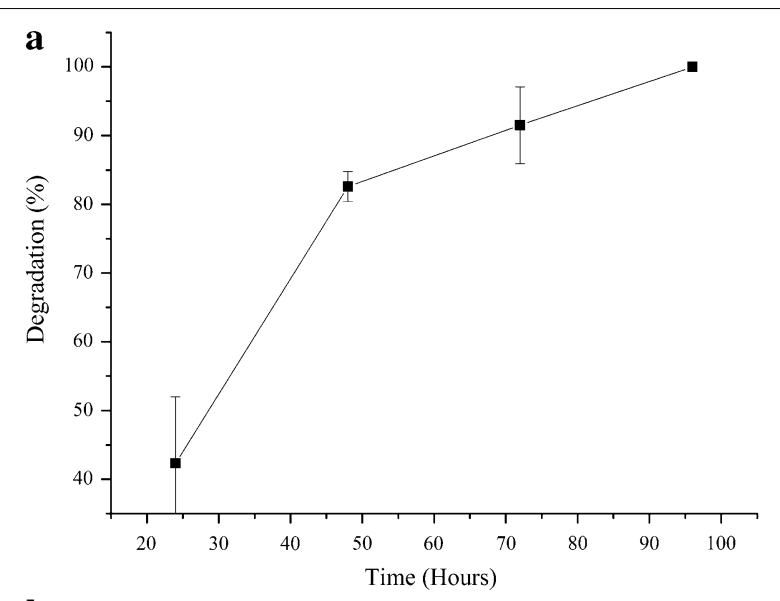

b

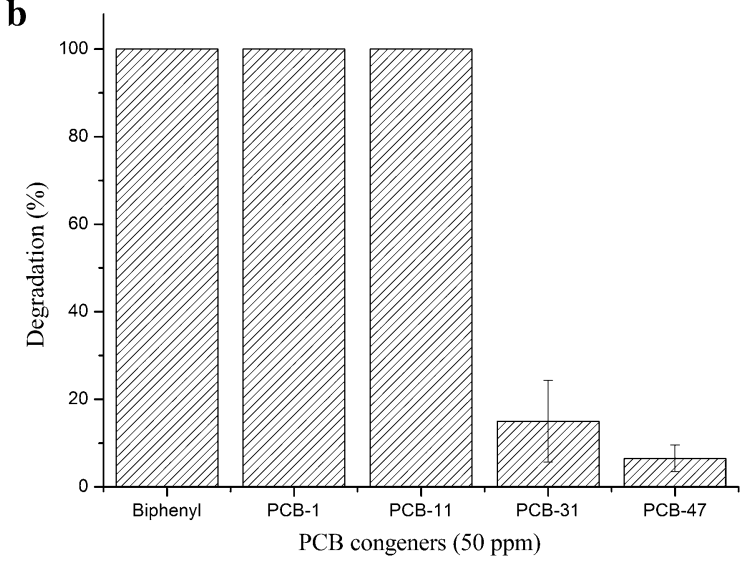

Fig. 3 A figure showing a the ability of YC-RL2 to degrade biphenyl (BP), $\mathbf{b}$ ability of YC-RL2 to degrade selected PCB congeners (50 ppm). The reaction was setup using TEM medium using BP, PCB congeners as the sole carbon and energy source in each case. The inoculum was $1 \%(V / V)$ of the medium. After 7 days of incubation (for PCB congener experiments), the residues were quantified using HPLC. Each setup was done in triplicate and error bars represent standard deviation from the mean value

\section{Diversity of dioxygenases}

A total of 22 dioxygenases were annotated in the genome. These sequences were checked with interpro, CDD and SMART databases to filter out false positives. Conserved domains as predicted by SMART and CDD are shown in Additional file 1: Table S1. Two sequences annotated as nitropropane dioxygenases were not considered for further analysis as that classification has been rendered obsolete and are currently classified as nitronate monooxygenases. The sequences were aligned using BlastP in the swissprot and pdb databases and retrieved sequences were used to construct a phylogenetic tree by MEGA 6.0 software and further annotated by iTOL (Letunic and Bork 2016). The phylogenetic tree showed that dioxygenases are clustered as shown in Fig. 6. Most dioxygenases were classified as ring cleaving or hydroxylating based on the swissprot or pdb and conserved domain database.

\section{Secondary metabolite biosynthesis potential}

To predict anabolic potential of strain YC-RL2, putative biosynthetic gene clusters (BGCs) were annotated by antiSMASH (antibiotics \& Secondary Metabolite Analysis Shell 4.0) (Weber et al. 2018). A total of 53 gene clusters were predicted; 6 non-ribosomal peptide synthetases (Nrps), 2 terpene, 1 arylpolyene, 1ectoine, 1 Type 1 polyketide synthases (T1pks), 1 bacteriocin, 1 fatty acid and 39 putative gene clusters (Additional file 1: Table S2). Such similar gene clusters were reported in G. lacunae $\mathrm{BS} 2^{\mathrm{T}}$ but have not been experimentally determined (Durrell et al. 2017).

\section{Heavy metal resistance}

The genome of strain YC-RL2 contains putative genes for heavy metal transport and resistance such as mercury (II) reductase, copper resistance proteins CopD and CopC, arsenical-resistance protein and tellurium resistance protein TerC. Amino acid sequences of genome derived metal resistance genes were searched against BacMet (Antibacterial Biocide and Metal Resistance Genes Database) using default parameters (Pal et al. 2014). The putative genes are located on genomic islands as predicted by IslandViewer 4 (Bertelli et al. 2017) (data not shown).

The strain was tested for growth in and tolerance of $1 \mathrm{mM}$ of $\mathrm{Cd}^{2+}, \mathrm{Co}^{2+}, \mathrm{Cu}^{2+}, \mathrm{Ni}^{2+}, \mathrm{Zn}^{2+}, \mathrm{Mn}^{2+}$ and $\mathrm{Pb}^{2+}$ ions and $0.1 \mathrm{mM}$ of $\mathrm{Hg}^{2+}$ and the result is shown in Fig. 7. The strain was tolerant to heavy metals tested except $\mathrm{Hg}^{2+}$.

\section{Discussion}

From the results of $16 \mathrm{~S}$ rRNA alignment and ANI calculation, strain YC-RL2 could be classified as G. alkanivorans. With a plethora of prokaryotic genomes in public databases, genomic classification was proposed by Chun et al. (2018) as the most reproducible and reliable method to infer phylogenetic relationships among prokaryotes (Chun et al. 2018).

The aerobic degradation pathway of PAEs in bacteria is initiated by the hydrolysis of ester bond to form corresponding MAPs. MAPs are further hydrolyzed to form PA (Liang et al. 2008) and esterases are the key enzymes (Luo et al. 2012). PA is then completely mineralized via protocatechuate (Benjamin et al. 2015) which is a central intermediate of aromatic ring degradation.

A previous study involving strain YC-RL2 showed that the strain could degrade di-2-ethylhexyl phthalate (DEHP) to PA and then BA instead of protocatechuate (Nahurira et al. 2017). More so, a phthalate degradation 


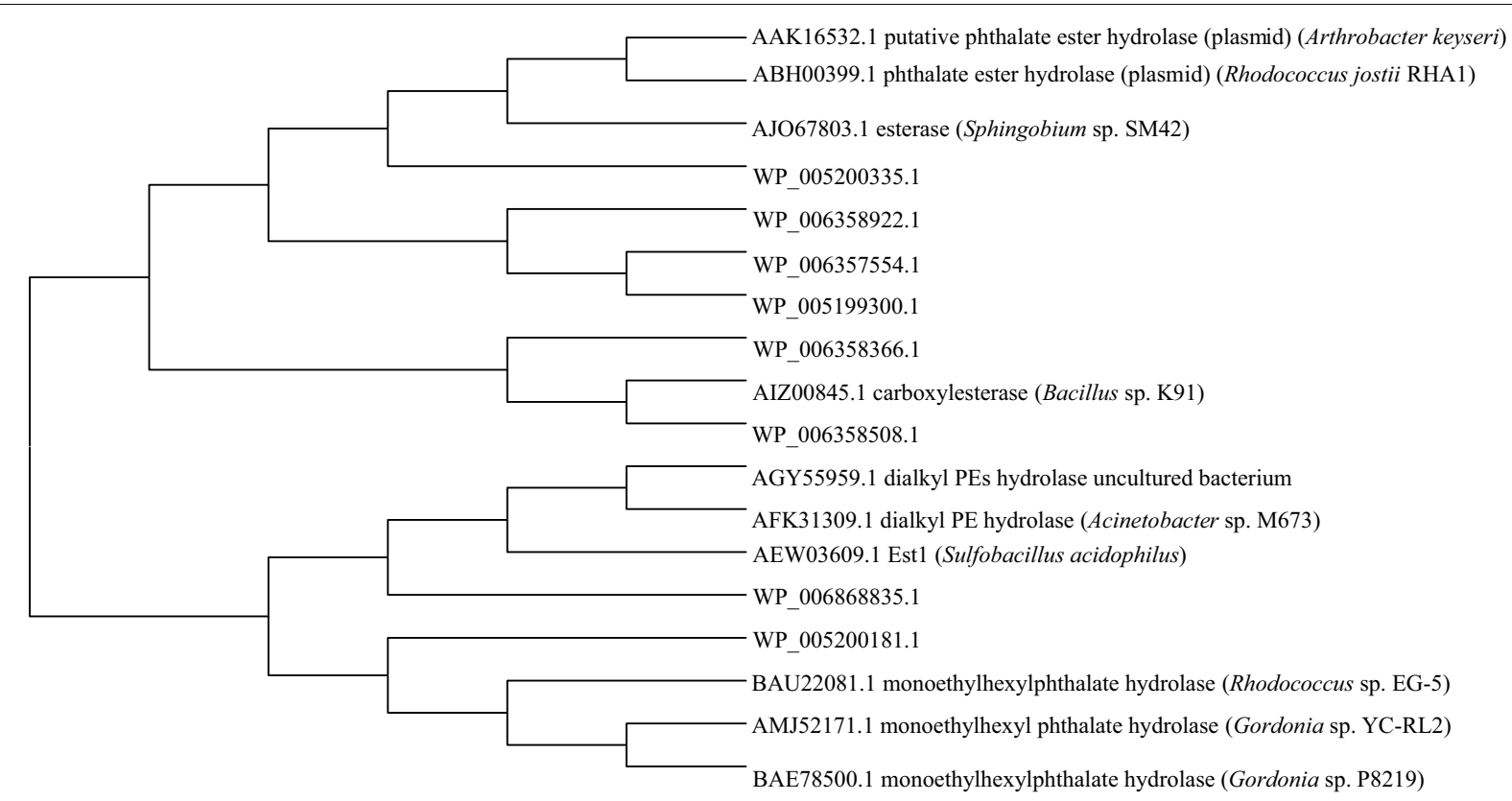

Fig. 4 A phylogenetic tree showing the relationship between selected putative esterases/hydrolases in YC-RL2 genome and reported phthalate hydrolases. The evolutionary history was inferred using the neighbor-joining algorithm. The bootstrap consensus tree inferred from 1000 replicates is taken to represent the evolutionary history of the taxa analyzed. The codes used for the sequences are Refseq accession numbers as annotated by $\mathrm{NCBI}$

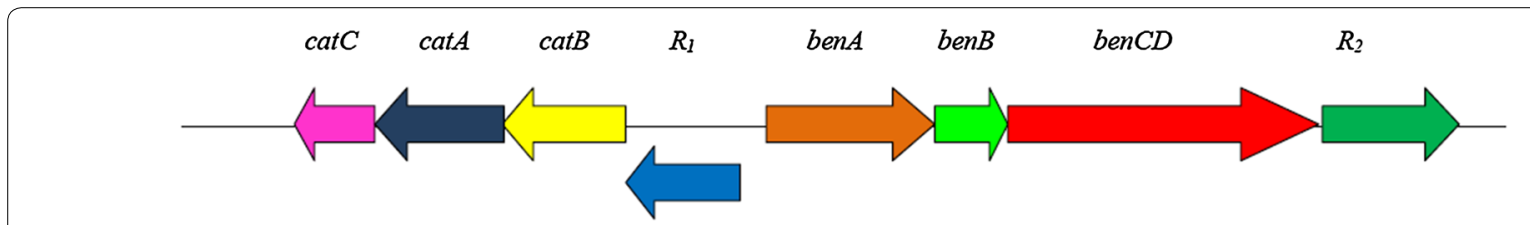

Fig. 5 A figure showing the arrangement of benzoate (benABCDR $)$ and catechol (catCABR $)$ operons in strain YC-RL2 (Not drawn to scale) as annotated by RAST

operon could not be identified in the genome of YC-RL2. We therefore hypothesized that PA could be decarboxylated to BA. Similar pathways were reported in Microbacterium sp. CQ0110Y (Chen et al. 2007), Mycobacterium sp. YC-RL4 (Ren et al. 2016). Thus the genome of strain YC-RL2 would have benzoate and catechol degradation clusters. We therefore analyzed the diversity of esterases, dioxygenases and decarboxylases. The putative esterases (Fig. 4) clustered majorly into two groups though the group containing CarEW from Bacillus sp K91was distinct. This is the only reported PAE esterase capable of hydrolyzing two bonds. This group contained WP_006358366.1 and WP_006358508.1 both annotated as carboxylesterases.

In typical phthalate degradation, further steps are accomplished by dioxygenases, dehydrogenases and later decarboxylases. A total of 22 dioxygenases were annotated in the genome and the phylogenetic tree between related dioxygenases shows that they are clustered into two major groups; Group I and II. The benzene ring is the most widely spread and thermodynamically stable chemical moiety which leads to its persistence in nature. Therefore, many aromatic compounds are major environmental pollutants (Díaz 2004). Ring cleavage is a crucial step in the aerobic degradation of aromatic pollutants and this is effected by oxygenases especially dioxygenases. Aromatic ring hydroxylating dioxygenases incorporate two atoms of molecular oxygen into their substrates to form cis-1, 2-dihydroxycyclohexadiene intermediates that are further acted upon by cis-diol dehydrogenases. This group of dioxygenases includes toluene, benzene, phthalate, naphthalene and biphenyl dioxygenases which are very important in xenobiotics degradation. Ringcleaving dioxygenases on the other hand, catalyze fission of catecholic compounds. No dioxygenases showed similarity to reported phthalate dioxygenases and a complete 


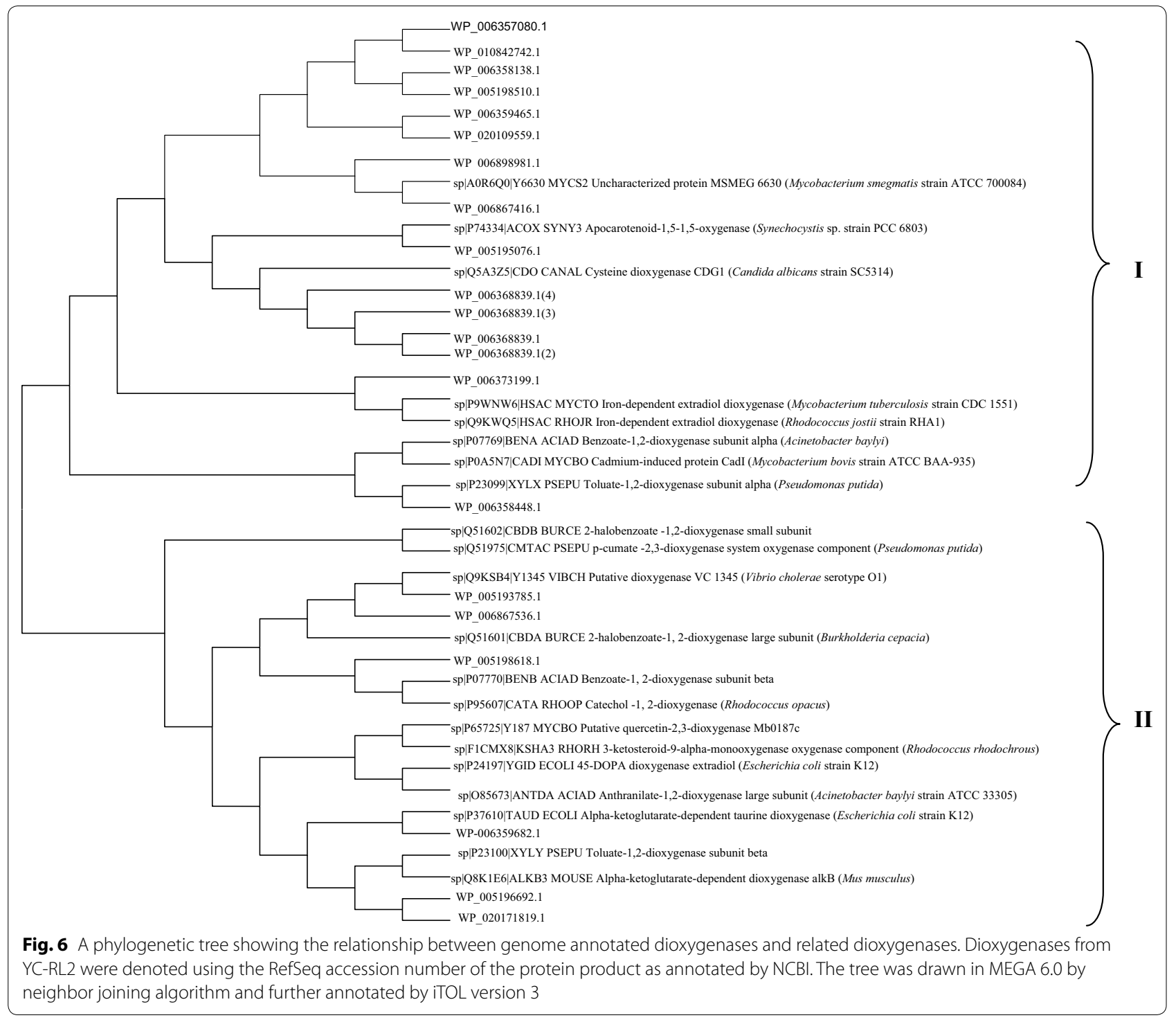

phthalate operon could not be annotated in the genome. This shows that strain YC-RL2 possesses a unique pathway of phthalate degradation that needs to be explored further.

There were complete catechol and benzoate gene clusters annotated in the genome. The gene organization of YC-RL2 benABCD operon differs from that of most reported ben $A B C D$ operons (Ezezika et al. 2006; Kitagawa et al. 2001; Zhan et al. 2008). The genes benC and ben $D$ are fused together with a $C$-terminal ligand binding domain (FCD) (Additional file 1: Figure S3) characteristic of GntR regulatory proteins. This domain is found in regulators of sugar biosynthesis operons that bind DNA through a helix-turn-helix (HTH) motif. There are two regulator coding genes; one upstream $\left(R_{1}\right)$ and other downstream $\left(R_{2}\right)$. The regulator $\mathrm{R}_{1}$ belongs to LsyR regulators while $R_{2}$ belongs to the LuxR unlike most LsyR regulators common to catechol and benzoate catabolism operons (Collier et al. 1998; Ezezika et al. 2006). LuxR regulators have a characteristic alpha-helical protein domains fold with sequence-specific DNA binding domains. $\mathrm{R}_{1}$ showed $35 \%$ identity to ben and cat operon transcriptional regulator from Acinetobacter baumannii (SCZ15558.1) (Zhan et al. 2008), 37\% to uncharacterized HTH-type transcriptional regulator YnfL (P77559.1) (Aiba et al. 1996) and 33\% to HTH-type transcriptional regulator BenM (O68014.2) (Collier et al. 1998). $R_{2}$ also showed $59 \%$ to HTH type of regulators Rv0890c (P9WMG1.1) and Mb0914c (P59969.1) which are mycobacterial proteins. 


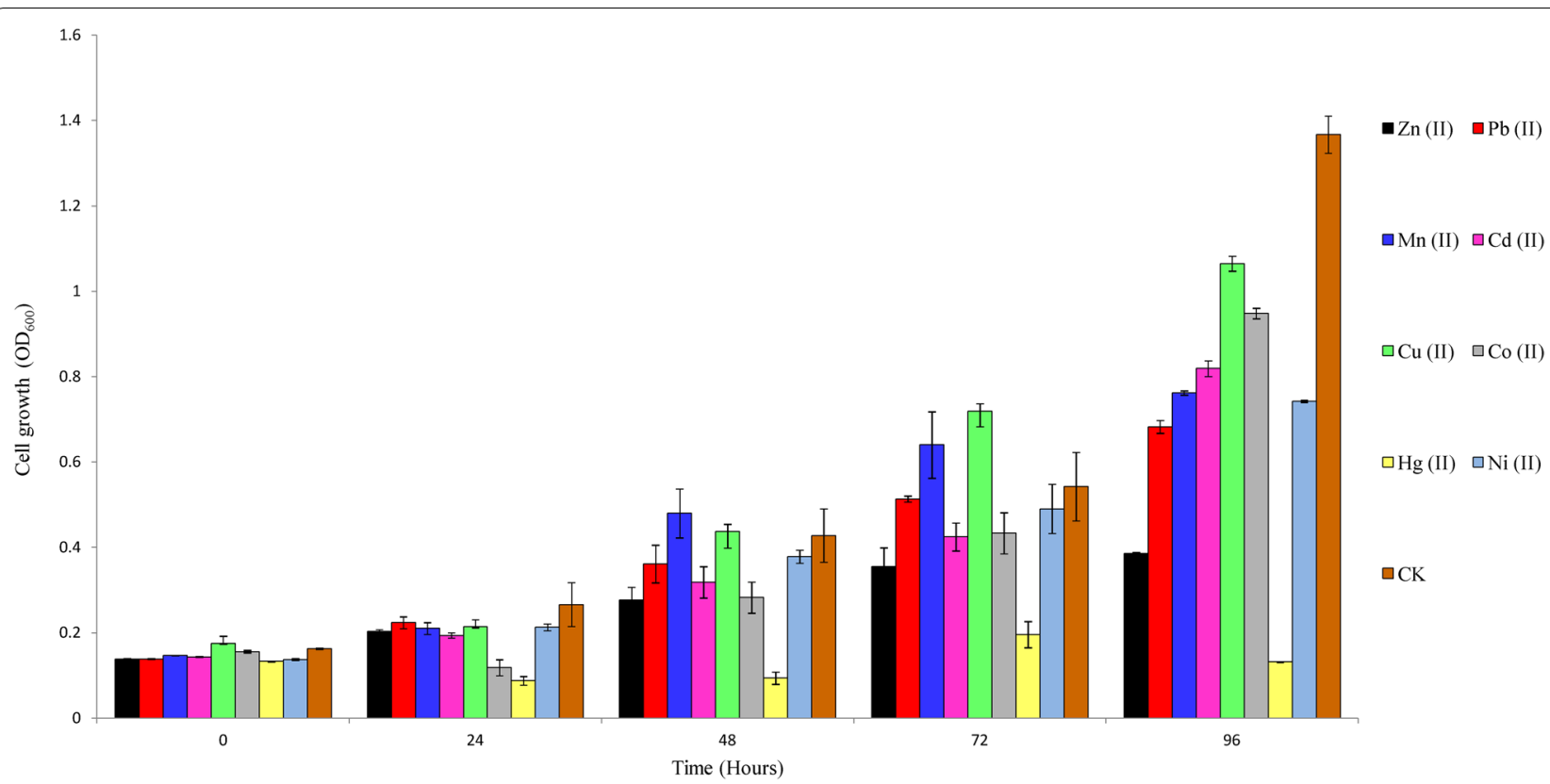

Fig. 7 A graph showing tolerance of strain YC-RL2 to heavy metal ions. The strain was inoculated in LB liquid medium supplemented with and tolerance of $1 \mathrm{mM}$ of $\mathrm{Cd}^{2+}, \mathrm{Co}^{2+}, \mathrm{Cu}^{2+}, \mathrm{Ni}^{2+}, \mathrm{Zn}^{2+}, \mathrm{Mn}^{2+}$ and $\mathrm{Pb}^{2+}$ ions and $0.1 \mathrm{mM}$ of $\mathrm{Hg}^{2+}$. Two controls were set up for each treatment. The setup with metal ions but no inoculum was considered as negative control and was used as a blank measurement. The inoculum without metal ions was considered as the positive control and as is denoted as CK. The experiment was done in triplicate for each heavy metal ion tested. The mean values were used to plot the graph and error bars represent standard deviation from the mean

In addition to degradation of xenobiotics, Gordonia members are known to be a source of natural products. A total of 53 putative BGCs were predicted in the genome. Predicted Nrps clusters shared little similarity to known gene clusters such as chartreusin (15\%), cahuitamycins (12\%), kanamycin (2\%), echosides (11\%). Predicted terpene clusters showed $6 \%$ and $62 \%$ similarity with SF2575 and sioxanthin biosynthetic gene clusters respectively. Sioxanthin is a class of glycosylated carotenoids reported in the genus of Salinispora; marine actinomycetes (Richter et al. 2015). Although the genus of Gordonia is well known for carotenoid biosynthesis, no clusters were annotated as carotenoid BGCs. However, 1 arylpolyene and 1Type I PKS cluster were predicted. Arylpolyene are class of pigments that are functionally similar to carotenoids and protect the bacterium from reactive oxidation (Schöner et al. 2016). Arylpolyenes are polyketide derivatives each contain a terminal aryl moiety connected to a polyene carboxylic acid. To our knowledge, arylpolyene production has not been experimentally proven but was also predicted in G. lacunae $\mathrm{BS}^{\mathrm{T}}$ (Durrell et al. 2017).

Annotated ectoine cluster shared $75 \%$ similarity to BGC0000853_c1 ectoine cluster. The predicted ectoine cluster comprised of acetyl glutamate-5-kinase, TetR family transcriptional regulator, haloalkane dehalogenase, ectoine synthase, aminotransferase class III and
GCN5 related-acetyltransferase genes. Ectoine is one many compatible solutes that protect and stabilizes cell membranes, proteins and DNA against drying and high temperatures, high osmolarity, and even pollutants (Bursy et al. 2008; Graf et al. 2008; Nakayama et al. 2000; Schnoor et al. 2004). In addition to ectoine biosynthesis, more genes coding for betaine, glycine and trehalose synthesis and transport were predicted in the genome. This explains the halotolerant nature of strain YC-RL2.

Some of the BGCs annotated as putative shared similarity with known BGCs for instance bacillomycin (20\%), coelichelin $(27 \%)$, nataxazole $(7 \%)$, rifamycin $(3 \%)$ and paromomycin $(5 \%)$. The ability to produce diversified secondary metabolites would not only enable the strain to survive in different ecological niches but also be harnessed for therapeutical, industrial and pharmaceutical uses.

Metals have a central role in the life processes of microorganisms. Some metals such as copper, iron, and zinc are essential in biochemical, structural and osmotic processes (Sterritt and Lester 1980). However, high metal concentration can be detrimental to microorganisms by displacement of essential metals from their native binding sites or through ligand interactions. Bacteria adapt to metals through a variety of genome-mediated resistance systems (Hassen et al. 1998; Tchounwou et al. 2012). 
Some Gordonia strains such as Gordonia sp WQ-01A, G. amicalis HS-11 and G. amarae MTCC 4818 are known to reduce some metals by formation of nanoparticles (Sowani et al. 2018).

The present study reveals that strain is metabolically versatile and has a high potential for secondary metabolites can be harnessed for various clinical and industrial applications. Presence of diverse dioxygenases, esterase/ hydrolase and metal resistant genes provide insight into the ability of this strain to survive in varied ecological niches. Cloning and expression of esterases involved in PAEs hydrolysis is currently underway. Based on these results, strain YC-RL2 genome will provide a rich genetic resource for further biotechnological and remediation studies.

\section{Additional file}

Additional file 1: Figure S1. A phylogenetic tree showing the relationship between 16S rDNA of strain YC-RL2 and 16S rDNA of validly published Gordonia species. The evolutionary history was inferred using the Neighbor-Joining algorithm. The bootstrap consensus tree inferred from 1000 replicates is taken to represent the evolutionary history of the taxa analyzed. Figure S2. Genome visualization by Circos. Figure S3. The conserved domains of putative benCD as predicted by NCBI conserved domain database (https://www.ncbi.nlm.nih.gov/cdd). Table S1. The Table showing conserved domains putative dioxygenases in YC-RL2 genome as predicted by SMART and CDD databases. Table S2. The table showing BGCs prediction based on the genome sequence of YC-RL2.

\section{Authors' contributions}

RN and $Y Y$ conceptualized research, designed experiments and wrote the manuscript; JW, YJ and SF helped in doing experiments especially genome sequencing, IK and AE helped in critically proof reading the manuscript. All authors approved the final version of the manuscript for publication and are accountable for all aspects of this work. All authors read and approved the final manuscript.

\section{Acknowledgements}

We acknowledge the intellectual and material contributions by Organization of Women in Science in the Developing world (Fund Reservation No. 3240266458) and SIDA (Swedish International Development Cooperation Agency).

\section{Competing interests}

The authors declare that they have no competing interests.

\section{Availability of data and materials}

The dataset supporting the conclusions of this article is included within the article. All data are fully available without restriction.

\section{Consent for publication}

Not applicable.

\section{Ethics approval and consent to participate}

This article does not contain any studies with human participants or animals.

\section{Funding}

This work was supported by the National Natural Science Foundation of China (Nos. 31540067 and 21876201), and the Basic Research Fund of CAAS (Nos. 0042012003, 0042014011 and 1610042017001).

\section{Publisher's Note}

Springer Nature remains neutral with regard to jurisdictional claims in published maps and institutional affiliations.

Received: 27 December 2018 Accepted: 3 January 2019

Published online: 04 February 2019

\section{References}

Aiba H, Baba T, Hayashi K, Inada T, Isono K, Itoh T, Kasai H, Kashimoto K, Kimura S, Kitakawa M, Kitagawa M, Making K, Miki T, Mizobuchi K, Mori H, Mori T, Motomura K, Nakade S, Nakamura Y, Nashimoto H, Nishio Y, Oshima T, Saito N, Sampei GI, Seki Y, Sivasundaram S, Tagami H, Takeda Jl, Takemoto K, Takeuchi Y, Wada C, Yamamoto Y, Horiuchi T (1996) A 570-kb DNA sequence of the Escherichia coli K-12 genome corresponding to the 28.0-40.1 min region on the linkage map. DNA Res. https:// doi.org/10.1093/dnares/3.6.363

Ardui S, Ameur A, Vermeesch JR, Hestand MS (2018) Single molecule real-time (SMRT) sequencing comes of age: applications and utilities for medical diagnostics. Nucleic Acids Res. https://doi.org/10.1093/nar/gky066

Arenskötter M, Bröker D, Steinbüchel A (2004) Biology of the metabolically diverse genus Gordonia. Appl Environ Microbiol 70:3195-3204. https:// doi.org/10.1128/AEM.70.6.3195-3204.2004

Aziz RK, Bartels D, Best A, DeJongh M, Disz T, Edwards RA, Formsma K, Gerdes S, Glass EM, Kubal M, Meyer F, Olsen GJ, Olson R, Osterman AL, Overbeek RA, McNeil LK, Paarmann D, Paczian T, Parrello B, Pusch GD, Reich C, Stevens $\mathrm{R}$, Vassieva O, Vonstein V, Wilke A, Zagnitko O (2008) The RAST server: rapid annotations using subsystems technology. BMC Genomics. https:// doi.org/10.1186/1471-2164-9-75

Benjamin S, Pradeep S, Josh MS, Kumar S, Masai E, Sarath Josh M, Kumar S, Masai E (2015) A monograph on the remediation of hazardous phthalates. J Hazard Mater 298:58-72. https://doi.org/10.1016/j.jhazm at.2015.05.004

Bertelli C, Laird MR, Williams KP, Lau BY, Hoad G, Winsor GL, Brinkman FSL (2017) IslandViewer 4: expanded prediction of genomic islands for largerscale datasets. Nucleic Acids Res. https://doi.org/10.1093/nar/gkx343

Brettin T, Davis JJ, Disz T, Edwards RA, Gerdes S, Olsen GJ, Olson R, Overbeek R, Parrello B, Pusch GD, Shukla M, Thomason JA, Stevens R, Vonstein V, Wat$\operatorname{tam}$ AR, Xia F (2015) RASTtk: a modular and extensible implementation of the RAST algorithm for building custom annotation pipelines and annotating batches of genomes. Sci Rep. https://doi.org/10.1038/srep08365

Bursy J, Kuhlmann AU, Pittelkow M, Hartmann H, Jebbar M, Pierik AJ, Bremer E (2008) Synthesis and uptake of the compatible solutes ectoine and 5-hydroxyectoine by Streptomyces coelicolor A3(2) in response to salt and heat stresses. Appl Environ Microbiol. https://doi.org/10.1128/aem.00768 $-08$

Chen JAA, Li X, Li J, Cao J, Qiu Z, Zhao Q, Xu C, Shu W (2007) Degradation of environmental endocrine disruptor di-2-ethylhexyl phthalate by a newly discovered bacterium, Microbacterium sp. strain CQ0110Y. Appl Microbiol Biotechnol 74:676-682. https://doi.org/10.1007/s00253-006-0700-3

Chun J, Oren A, Ventosa A, Christensen H, Arahal DR, Costa MS, Rooney AP, Yi $H, X u X$, De Meyer S, Trujillo ME (2018) Proposed minimal standards for the use of genome data for the taxonomy of prokaryotes. Int J Syst Evol Microbiol. https://doi.org/10.1099/ijsem.0.002516

Collier LS, Gaines GL, Neidle EL (1998) Regulation of benzoate degradation in Acinetobacter sp. strain ADP1 by BenM, a LysR-type transcriptional activator. J Bacteriol 180(9):2493-2501

Das N, Chandran P (2011) Microbial degradation of petroleum hydrocarbon contaminants: an overview. Biotechnol Res Int. https://doi. org/10.4061/2011/941810

Díaz E (2004) Bacterial degradation of aromatic pollutants: a paradigm of metabolic versatility. Int Microbiol 7:173-180

Ding J, Wang C, Xie Z, Li J, Yang Y, Mu Y, Tang X, Xu B, Zhou J, Huang Z (2015) Properties of a newly identified esterase from Bacillus sp. K91 and its novel function in diisobutyl phthalate degradation. PLOS ONE 10:e0119216. https://doi.org/10.1371/journal.pone.0119216

Drzyzga O (2012) The strenghts and weaknesses of Gordonia: a review of an emerging genus with increasing biotechnological potential. Crit Rev Microbiol 38:300-316. https://doi.org/10.3109/1040841X.2012.668134 
Durrell K, Prins A, Le Roes-Hill M (2017) Draft genome sequence of Gordonia lacunae BS2T. Genome Announc. https://doi.org/10.1128/genom ea.00959-17

Ebenau-Jehle C, Mergelsberg M, Fischer S, Brüls T, Jehmlich N, Von Bergen M, Boll M (2017) An unusual strategy for the anoxic biodegradation of phthalate. ISME J. https://doi.org/10.1038/ismej.2016.91

El-Shahawi MS, Hamza A, Bashammakh AS, Al-Saggaf WT (2010) An overview on the accumulation, distribution, transformations, toxicity and analytical methods for the monitoring of persistent organic pollutants. Talanta. https://doi.org/10.1016/j.talanta.2009.09.055

Ezezika OC, Collier-Hyams LS, Dale HA, Burk AC, Neidle EL (2006) CatM regulation of the benABCDE operon: functional divergence of two LysR-type paralogs in Acinetobacter baylyi ADP1. Appl Environ Microbiol. https://doi. org/10.1128/aem.72.3.1749-1758.2006

Fu F, Wang Q (2011) Removal of heavy metal ions from wastewaters: a review. J Environ Manage. https://doi.org/10.1016/j.jenvman.2010.11.011

Graf R, Anzali S, Buenger J, Pfluecker F, Driller H (2008) The multifunctional role of ectoine as a natural cell protectant. Clin Dermatol. https://doi. org/10.1016/j.clindermatol.2008.01.002

Hassen A, Saidi N, Cherif M, Boudabous A (1998) Resistance of environmental bacteria to heavy metals. Bioresour Technol. https://doi.org/10.1016/ s0960-8524(97)00161-2

Hausmann S, Jaeger K-E (2010) Lipolytic enzymes from bacteria. In: Handbook of hydrocarbon and lipid microbiology. Springer, Berlin, Heidelberg, pp 1099-1126

Iwata M, Imaoka T, Nishiyama T, Fujii T (2015) Re-characterization of mono2-ethylhexyl phthalate hydrolase belonging to the serine hydrolase family. J Biosci Bioeng. https://doi.org/10.1016/j.jbiosc.2016.01.008

Jayakumar V, Sakakibara Y (2017) Comprehensive evaluation of non-hybrid genome assembly tools for third-generation PacBio long-read sequence data. Brief Bioinform. https://doi.org/10.1093/bib/bbx147

Kanehisa M, Goto S, Kawashima S, Okuno Y (2018) The KEGG resource for deciphering the genome. Nucleic Acids Res. https://doi.org/10.1093/nar/ gkh063

Kitagawa W, Miyauchi K, Masai E, Fukuda M (2001) Cloning and characterization of benzoate catabolic genes in the gram-positive polychlorinated biphenyl degrader Rhodococcus sp. strain RHA1. J Bacteriol. https://doi. org/10.1128/jb.183.22.6598-6606.2001

Krzywinski M, Schein J, Birol I, Connors J, Gascoyne R, Horsman D, Jones SJ, Marra MA (2009) Circos: an information aesthetic for comparative genomics. Genome Res. https://doi.org/10.1101/gr.092759.109

Letunic I, Bork P (2016) Interactive tree of life (iTOL) v3: an online tool for the display and annotation of phylogenetic and other trees. Nucleic Acids Res. https://doi.org/10.1093/nar/gkw290

Letzel AC, Pidot SJ, Hertweck C (2014) Genome mining for ribosomally synthesized and post-translationally modified peptides (RiPPs) in anaerobic bacteria. BMC Genomics. https://doi.org/10.1186/1471-2164-15-983

Liang DW, Zhang T, Fang HHP, He J (2008) Phthalates biodegradation in the environment. Appl Microbiol Biotechnol 80:183-198. https://doi. org/10.1007/s00253-008-1548-5

Liu L, Hao T, Xie Z, Horsman GP, Chen Y (2016) Genome mining unveils widespread natural product biosynthetic capacity in human oral microbe Streptococcus mutans. Sci Rep. https://doi.org/10.1038/srep37479

Luo ZH, Pang KL, Wu YR, Gu JD, Chow RK, Vrijmoed LL (2012) Degradation of phthalate esters by Fusarium sp. DMT-5-3 and Trichosporon sp. DMI-5-1 isolated from mangrove sediments. Prog Mol Subcell Biol 53:299-328. https://doi.org/10.1007/978-3-642-23342-5_15

Megharaj M, Ramakrishnan B, Venkateswarlu K, Sethunathan N, Naidu R (2011) Bioremediation approaches for organic pollutants: a critical perspective. Environ Int. https://doi.org/10.1016/j.envint.2011.06.003

Nahurira R, Ren L, Song J, Jia Y, Wang J, Fan S, Wang H, Yan Y (2017) Degradation of di(2-ethylhexyl) phthalate by a novel Gordonia alkanivorans strain YC-RL2. Curr Microbiol. https://doi.org/10.1007/s00284-016-1159-9

Nahurira R, Jia Y, Ren L, Wang J, Qiao C, Fan S, Wang H, Yan Y (2018) Biochemical and structural characterization of a monoethylhexyl phthalate hydrolase from Gordonia alkanivorans strain YC-RL2. Acta Microbiol Sin 58:303-313. https://doi.org/10.13343/j.cnki.wsxb.20170142
Nakayama H, Yoshida K, Ono H, Murooka Y, Shinmyo A (2000) Ectoine, the compatible solute of Halomonas elongata, confers hyperosmotic tolerance in cultured tobacco cells. Plant Physiol. https://doi.org/10.1104/ pp.122.4.1239

Pal C, Bengtsson-Palme J, Rensing C, Kristiansson E, Larsson DGJ (2014) BacMet: antibacterial biocide and metal resistance genes database. Nucleic Acids Res. https://doi.org/10.1093/nar/gkt1252

Ren L, Jia Y, Ruth N, Qiao C, Wang J, Zhao B, Yan Y (2016) Biodegradation of phthalic acid esters by a newly isolated Mycobacterium sp YC-RL4 and the bioprocess with environmental samples. Environ Sci Pollut Res. https:// doi.org/10.1007/s11356-016-6829-4

Richter TKS, Hughes CC, Moore BS (2015) Sioxanthin, a novel glycosylated carotenoid, reveals an unusual subclustered biosynthetic pathway. Environ Microbiol. https://doi.org/10.1111/1462-2920.12669

Schäfer RB, Von Der Ohe PC, Kühne R, Schüürmann G, Liess M (2011) Occurrence and toxicity of 331 organic pollutants in large rivers of north Germany over a decade (1994 to 2004). Environ Sci Technol. https://doi. org/10.1021/es2013006

Schneider K, Graf E, Irran E, Nicholson G, Stainsby FM, Goodfellow M, Borden SA, Keller S, Süssmuth RD, Fiedler HP (2008) Bendigoles A $\approx$ C, new steroids from Gordonia australis Acta 2299. J Antibiot (Tokyo). https://doi. org/10.1038/ja.2008.50

Schnoor M, Voß P, Cullen P, Böking T, Galla HJ, Galinski EA, Lorkowski S (2004) Characterization of the synthetic compatible solute homoectoine as a potent PCR enhancer. Biochem Biophys Res Commun. https://doi. org/10.1016/j.bbrc.2004.07.200

Schöner TA, Gassel S, Osawa A, Tobias NJ, Okuno Y, Sakakibara Y, Shindo K, Sandmann G, Bode HB (2016) Aryl polyenes, a highly abundant class of bacterial natural products are functionally related to antioxidative carotenoids. ChemBioChem. https://doi.org/10.1002/cbic.201500474

Shin SC, Ahn DH, Kim SJ, Lee H, Oh TJ, Lee JE, Park H (2013) Advantages of single-molecule real-time sequencing in high-GC Content genomes. PLOS ONE 1:1. https://doi.org/10.1371/journal.pone.0068824

Sowani H, Kulkarni M, Zinjarde S (2018) An insight into the ecology, diversity and adaptations of Gordonia species. Crit Rev Microbiol 44:393-413. https ://doi.org/10.1080/1040841X.2017.1418286

Sterritt RM, Lester JN (1980) Interactions of heavy metals with bacteria. Sci Total Environ. https://doi.org/10.1016/0048-9697(80)90122-9

Ta-Chen L, Chang JS, Young CC (2008) Exopolysaccharides produced by Gordonia alkanivorans enhance bacterial degradation activity for diesel. Biotechnol Lett. https://doi.org/10.1007/s10529-008-9667-8

Tamura K, Stecher G, Peterson D, Filipski A, Kumar S (2013) MEGA6: molecular evolutionary genetics analysis version 6.0. Mol Biol Evol 30(12):27252729. https://doi.org/10.1093/molbev/mst197

Tatusov RL (2000) The COG database: a tool for genome-scale analysis of protein functions and evolution. Nucleic Acids Res. https://doi.org/10.1093/ nar/28.1.33

Tchounwou PB, Yedjou CG, Patlolla AK, Sutton DJ (2012) Heavy metals toxicity and the environment. Mol Clin Environ Toxicol. https://doi. org/10.1007/978-3-7643-8340-4_6

Uhrynowski W, Decewicz P, Dziewit L, Radlinska M, Krawczyk PS, Lipinski L, Adamska D, Drewniak L (2017) Analysis of the genome and mobilome of a dissimilatory arsenate reducing Aeromonas sp. O23A reveals multiple mechanisms for heavy metal resistance and metabolism. Front Microbiol. https://doi.org/10.3389/fmicb.2017.00936

Wang S, Shi X (2001) Molecular mechanisms of metal toxicity and carcinogenesis. Mol Cell Biochem. https://doi.org/10.1023/a:1017918013293

Weber T, Blin K, Duddela S, Krug D, Kim HU, Bruccoleri R, Lee SY, Fischbach MA, Rolf M, Wohlleben W, Breitling R, Takano E, Medema MH (2018) AntiSMASH 3.0 - a comprehensive resource for the genome mining of biosynthetic gene clusters. Nucleic Acids Res 43:237-243. https://doi. org/10.1093/nar/gkv437

Whangsuk W, Sungkeeree P, Nakasiri M, Thiengmag S, Mongkolsuk S, Loprasert S (2015) Two endocrine disrupting dibutyl phthalate degrading esterases and their compensatory gene expression in Sphingobium sp. SM42. Int Biodeterior Biodegrad. https://doi.org/10.1016/j.ibiod.2014.12.006

Zhan Y, Yu H, Yan Y, Chen M, Lu W, Li S, Peng Z, Zhang W, Ping S, Wang J, Lin M (2008) Genes involved in the benzoate catabolic pathway 
in Acinetobacter calcoaceticus PHEA-2. Curr Microbiol. https://doi. org/10.1007/s00284-008-9251-4

Zhang XY, Fan X, Qiu YJ, Li CY, Xing S, Zheng YT, Xu JH (2014) Newly identified thermostable esterase from Sulfobacillus acidophilus: properties and performance in phthalate ester degradation. Appl Environ Microbiol. https:// doi.org/10.1128/aem.02072-14
Zhao HM, Hu RW, Du H, Xin XP, Li YW, Li H, Cai QY, Mo CH, Liu JS, Zhou DM, Wong MH, He ZL (2018) Functional genomic analysis of phthalate acid ester (PAE) catabolism genes in the versatile PAE-mineralising bacterium Rhodococcus sp. 2G. Sci Total Environ. https://doi.org/10.1016/j.scito tenv.2018.05.337

\section{Submit your manuscript to a SpringerOpen ${ }^{\circ}$ journal and benefit from:}

- Convenient online submission

- Rigorous peer review

- Open access: articles freely available online

- High visibility within the field

- Retaining the copyright to your article

Submit your next manuscript at $\boldsymbol{\nabla}$ springeropen.com 